\title{
Exact Solution of Electromagnetic Scattering From a Dipole Antenna Located Inside a Multilayer Metamaterial Oblate Spheroidal Cavity
}

\author{
Yangqing Liu, Tadahiro Negishi, and Danilo Erricolo
}

\begin{abstract}
A new exact for a solution half oblate spheroidal cavity filled with double-negative and double-positive metamaterials and surrounded by perfect electric conductor walls with a circular opening is considered. The structure is illuminated by a dipole source, either electric or magnetic, located on the axis of symmetry and axially oriented. Analytical expressions and numerical examples are provided.
\end{abstract}

\section{Introduction}

Analytical solutions of electromagnetic scattering problems provide an exact mathematical description of the scattered field, which is important to learn the relationship between a given geometrical shape and the resulting scattered field. In this context, we refer to exact analytical solutions as expressions obtained from the solution of electromagnetic boundary value problems for which it is possible to obtain an exact mathematical representation, typically in the form of the sum of an infinite series involving an appropriate class of functions. In particular, the expansion coefficients of the series terms must be known a priori and not be the result of the evaluation of an infinite system of equations. The numerical computation of the sum of the series may benefit from the application of acceleration methods [1]. A brief literature survey finds that, in addition to the known exact solutions published in [2], the introduction of the isorefractive condition by Uslenghi [3, 4] allowed for the development of many new exact solutions. In particular, new geometries involving isorefractive materials are available for scattering from infinite bodies, such as shapes involving the 2D wedge [5-7], the elliptical cylinder [8-18], the paraboloid [19-21], and finite bodies, such as shapes involving spheroidal geometries [22-25].

The new geometry considered in this article consists of a perfect electric conductor (PEC) ground plane with a circular hole as shown in Fig. 1. Flush

Manuscript received 16 December 2021.

Yangqing Liu is with the University of Illinois Chicago, 851 South Morgan Street, Chicago, Illinois 60607, USA; e-mail: yliu299@uic.edu.

Tadahiro Negishi was with the University of Illinois Chicago, 851 South Morgan Street, Chicago, Illinois 60607, USA; e-mail: t.negishi@gmail.com.

Danilo Erricolo is with the University of Illinois Chicago, 851 South Morgan Street, Chicago, Illinois 60607, USA; e-mail: derric1@uic.edu. mounted underneath the hole, there is a PEC wall, shaped as a half-oblate spheroid, which determines a cavity. Inside the cavity there are two layers, one made of ordinary double-positive (DPS) material with positive dielectric permittivity and positive magnetic permeability and the other made of double-negative (DNG) metamaterial with negative dielectric permittivity and negative magnetic permeability. The interface between the two layers is an oblate spheroidal surface. The material outside the cavity and above the ground plane is DPS. The DPS and DNG materials are anti-isorefractive to each other. Preliminary results were presented in [26]. A related geometry with the cavity filled with isorefractive material was investigated in [22]. The cavity filled with DNG metamaterial was investigated in [27], while a cavity with two layers but with the dipole source located outside of the cavity was investigated in [25]. Exact analytic solutions for a dipole source located inside either one of the layers in the cavity, along the axis of symmetry of the structure and axially oriented, can be obtained. The solutions are expressed in terms of infinite series containing oblate spheroidal functions, according to the notation and properties given in [28] and [29]. The analytical solution is expressed in the phasor domain, where the time dependence $\mathrm{e}^{i \omega t}$ is assumed and suppressed throughout. The relation between Cartesian coordinates $(x, y, z)$ and oblate spheroidal coordinates $(\eta, \xi$, $\varphi)$ is

$$
\begin{gathered}
x=\frac{d}{2} \sqrt{\left(1+\xi^{2}\right)\left(1-\eta^{2}\right)} \cos \varphi \\
y=\frac{d}{2} \sqrt{\left(1+\xi^{2}\right)\left(1-\eta^{2}\right)} \sin \varphi \\
z=\frac{d}{2} \xi \eta
\end{gathered}
$$

with $0 \leq \xi<\infty,-1 \leq \eta \leq 1$, and $0 \leq \varphi \leq 2 \pi$.

\section{Electric Dipole}

For an electric dipole located along the $z$-axis at $\left(\xi_{0}<\xi_{1}, \eta_{0}=-1\right)$, corresponding to an incident electric Hertz vector $\pm \hat{z} \exp (i k R) /(k R)$, the incident magnetic field is oriented in the $\varphi$-direction. The positive (negative) sign applies when the dipole is located inside a DPS (DNG) material [29]. In the absence of the cavity, the $\varphi$-component of the total field, that is, the sum of the incident field and the field reflected by the infinite metal plate at $z=0$, is 


\section{DPS}

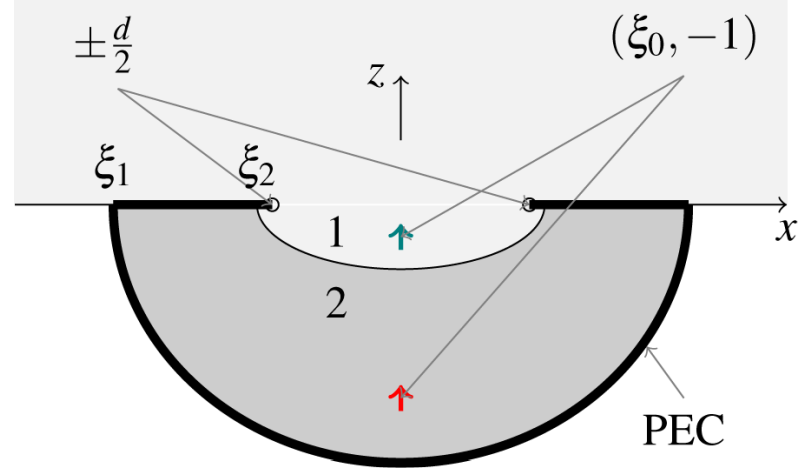

Figure 1. Sample problem geometry: layer 1 is filled with DPS metamaterial and layer 2 with DNG material.

$$
\begin{aligned}
& \left(H_{\varphi}^{i}+H_{\varphi}^{r}\right)_{D P S} \\
& =\frac{-4 i k^{2}}{Z_{p} \sqrt{\xi_{0}^{2}+1}} \sum_{l=0}^{\infty} \frac{(-1)^{l}}{\rho_{1,2 l+1}(-i c) N_{1,2 l+1}(-i c)} \\
& \quad \times R_{1,2 l+1}^{(1)}\left(-i c, i \xi_{<}\right) R_{1,2 l+1}^{(4)}\left(-i c, i \xi_{>}\right) S_{1,2 l+1}(-i c, \eta)
\end{aligned}
$$

where $R^{(1)}$ and $R^{(4)}$ are radial oblate spheroidal functions of the first and fourth kind, respectively; $S$ are the angular oblate spheroidal functions; $\rho$ and $N$ are normalization factors; $Z_{p}$ is the impedance in the DPS layer; and $c=k d / 2$. The symbol $<(>)$ refers to the smaller (greater) between $\xi$ and $\xi_{0}$. The perturbation due to the cavity adds a diffracted field through the circular hole, given in the region $z>0$ by

$$
\begin{aligned}
H_{\varphi, D P S}^{d}= & \frac{-4 i k^{2}}{Z_{p} \sqrt{\xi_{0}^{2}+1}} \sum_{l=0}^{\infty} \frac{(-1)^{l} S_{1,2 l+1}(-i c, \eta)}{\rho_{1,2 l+1}(-i c) N_{1,2 l+1}(-i c)} \\
& \times a_{l} R_{1,2 l+1}^{(4)}(-i c, i \xi)
\end{aligned}
$$

with an unknown coefficient $a_{1}$. In the DPS layer, the scattered magnetic field takes the form

$$
\begin{aligned}
H_{\varphi, D P S}^{s}= & \frac{-4 i k^{2}}{Z_{p} \sqrt{\xi_{0}^{2}+1}} \sum_{l=0}^{\infty} \frac{(-1)^{l} S_{1,2 l+1}(-i c, \eta)}{\rho_{1,2 l+1}(-i c) N_{1,2 l+1}(-i c)} \\
& \times\left[b_{l} R_{1,2 l+1}^{(1)}(-i c, i \xi)+c_{l} R_{1,2 l+1}^{(4)}(-i c, i \xi)\right]
\end{aligned}
$$

with unknown coefficients $b_{1}$ and $c_{1}$. In the DNG layer, the scattered magnetic field due to the cavity takes the form of

$$
\begin{aligned}
H_{\varphi, D N G}^{s}= & \frac{-4 i k^{2}}{Z_{n} \sqrt{\xi_{0}^{2}+1}} \sum_{l=0}^{\infty} \frac{(-1)^{l} S_{1,2 l+1}(i c, \eta)}{\rho_{1,2 l+1}(i c) N_{1,2 l+1}(i c)} \\
& \times\left[d_{l} R_{1,2 l+1}^{(1)}(i c, i \xi)+e_{l} R_{1,2 l+1}^{(4)}(i c, i \xi)\right]
\end{aligned}
$$

with unknown coefficients $d_{1}$ and $e_{1}$, and $Z_{n}$ is the impedance in the DNG layer. The unknown coefficients are obtained by enforcing the boundary conditions at $\xi=0, \xi=\xi_{2}$, and $\xi=\xi_{1}$,

$$
\begin{gathered}
\left.E_{\eta, 2}\right|_{\xi=\xi_{1}}=0 \\
\left.E_{\eta, 1}\right|_{\xi=0}+\left.E_{\eta}^{d}\right|_{\xi=0}=0 \\
\left.H_{\varphi, 1}\right|_{\xi=0}=\left.H_{\varphi}^{d}\right|_{\xi=0} \\
\left.E_{\eta, 1}\right|_{\xi=\xi_{2}}+\left.E_{\eta, 2}\right|_{\xi=\xi_{2}}=0 \\
\left.H_{\varphi, 1}\right|_{\xi=\xi_{2}}=\left.H_{\varphi, 2}\right|_{\xi=\xi_{2}}
\end{gathered}
$$

where $H$ and $E$ are the total fields, $H=H^{i}+H^{r}+H^{s}$ at $z$ $\leq 0$, and $H=H^{d}$ at $z \geq 0$.

\section{Magnetic Dipole}

Similar to the electric dipole source case, a magnetic dipole along the $z$-axis at $\left(\xi_{0}<\xi_{1}, \eta_{0}=-1\right)$ corresponds to an incident magnetic Hertz vector $\pm \hat{z}$ $\exp (i k R) /(k R)$ causing an incident electric field oriented in the $\varphi$-direction. In the absence of the cavity, the $\varphi$-component of the total field given by the sum of incident field and the field reflected by the infinite metal plate at $z=0$ is

$$
\begin{aligned}
\left(E_{\varphi}^{i}+\right. & \left.E_{\varphi}^{r}\right)_{D P S} \\
= & \frac{-8 k^{2}}{d Z_{p} \omega \epsilon_{p} \sqrt{\xi_{0}^{2}+1}} \\
& \times \sum_{l=0}^{\infty} \frac{(-1)^{l}}{\rho_{1,2 l+1}(-i c) N_{1,2 l+1}(-i c)} S_{1,2 l+1}(-i c, \eta) \\
& \times\left[R_{1,2 l+1}^{(1)}\left(-i c, i \xi_{<}\right) R_{1,2 l+1}^{(4)}\left(-i c, i \xi_{>}\right)\right]
\end{aligned}
$$

The perturbation due to the cavity through the circular hole is accounted for in the region $z>0$ by the diffracted electric field

$$
\begin{aligned}
E_{\varphi, D P S}^{d}= & \frac{-8 k^{2}}{d Z_{p} \omega \epsilon_{p} \sqrt{\xi_{0}^{2}+1}} \sum_{l=0}^{\infty} \frac{(-1)^{l}}{\rho_{1,2 l+1}(-i c) N_{1,2 l+1}(-i c)} \\
& \times a_{l} S_{1,2 l+1}(-i c, \eta) R_{1,2 l+1}^{(4)^{\prime}}(-i c, i \xi)
\end{aligned}
$$

In the DPS layer, the scattered field is

$$
\begin{aligned}
E_{\varphi, D P S}^{s}= & \frac{-8 k^{2}}{d Z_{p} \omega \epsilon_{p} \sqrt{\xi_{0}^{2}+1}} \sum_{l=0}^{\infty} \frac{(-1)^{l} S_{1,2 l+1}(-i c, \eta)}{\rho_{1,2 l+1}(-i c) N_{1,2 l+1}(-i c)} \\
& \times\left[b_{l} R_{1,2 l+1}^{(1)^{\prime}}(-i c, i \xi)+c_{l} R_{1,2 l+1}^{(4)^{\prime}}(-i c, i \xi)\right] \quad(9)
\end{aligned}
$$

while in the DNG layer, the scattered field is 


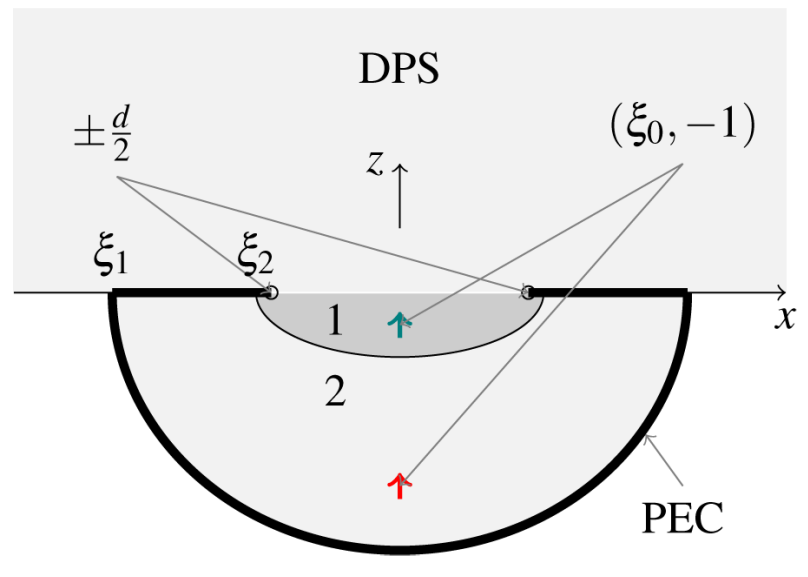

Figure 2. Sample problem geometry: layer 1 is filled with DNG metamaterial and layer 2 with DPS material.

$$
\begin{aligned}
E_{\varphi, D N G}^{s}= & \frac{-8 k^{2}}{d Z_{n} \omega \epsilon_{n} \sqrt{\xi_{0}^{2}+1}} \sum_{l=0}^{\infty} \frac{(-1)^{l} S_{1,2 l+1}(i c,-\eta)}{\rho_{1,2 l+1}(i c) N_{1,2 l+1}(i c)} \\
& \times\left[d_{l} R_{1,2 l+1}^{(1)^{\prime}}(i c, i \xi)+e_{l} R_{1,2 l+1}^{(4)^{\prime}}(i c, i \xi)\right]
\end{aligned}
$$

Again, to obtain the unknown coefficients, the following boundary conditions are enforced at $\xi=0, \xi=$ $\xi_{2}$, and $\xi=\xi_{1}$ :

$$
\begin{gathered}
\left.E_{\varphi, 2}\right|_{\xi=\xi_{1}}=0 \\
\left.E_{\varphi, 1}\right|_{\xi=0}+\left.E_{\varphi}^{d}\right|_{\xi=0}=0 \\
\left.H_{\eta, 1}\right|_{\xi=0}=\left.H_{\eta}^{d}\right|_{\xi=0} \\
\left.E_{\varphi, 1}\right|_{\xi=\xi_{2}}+\left.E_{\varphi, 2}\right|_{\xi=\xi_{2}}=0 \\
\left.H_{\eta, 1}\right|_{\xi=\xi_{2}}=\left.H_{\eta, 2}\right|_{\xi=\xi_{2}}
\end{gathered}
$$

and the total field $E=E^{i}+E^{r}+E^{s}$ in the region $z \leq 0$ and $E=E^{d}$ in the region $z \geq 0$.

\section{Numerical Results}

The numerical results for the total magnetic field by an electric dipole and for the total electric field by a magnetic dipole are obtained in MATLAB. Dipoles are placed at two locations inside the cavity: in layer 1 at $\left(\xi_{0}=\right.$ $\left.0.25, \eta_{0}=-1\right)$ (the green arrow in Figure 1) and in layer 2 at $\left(\xi_{0}=1.5, \eta_{0}=-1\right)$ (the red arrow in Figure 1$)$. The cavity material distribution has two cases: the DPS-DNG case with layer $1 \mathrm{DPS}$ and layer $2 \mathrm{DNG}$ and the DNGDPS case with layer $1 \mathrm{DNG}$ and layer 2 DPS. The oblate spheroidal cavity has boundaries at $\xi_{1}=2$ and $\xi_{2}=0.5$ when $c=1, d=2$, and $\zeta=\frac{Z_{D P S}}{Z_{D N G}}=0.5$. The magnitudes of the magnetic field due to an electric dipole, with incident electric Hertz vector $\pm \hat{z} \exp (i k R) /(k R)$, at various locations and for different material combinations are given in Figures 3-6. The magnitudes of the electric field

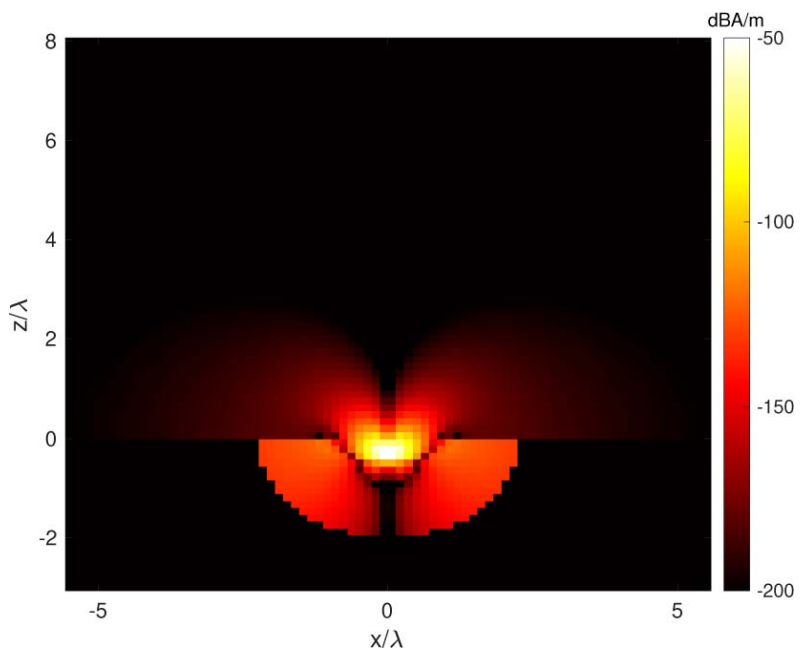

Figure 3. Magnitude of the total magnetic field due to an electric dipole in layer 1 for the DPS-DNG cavity.

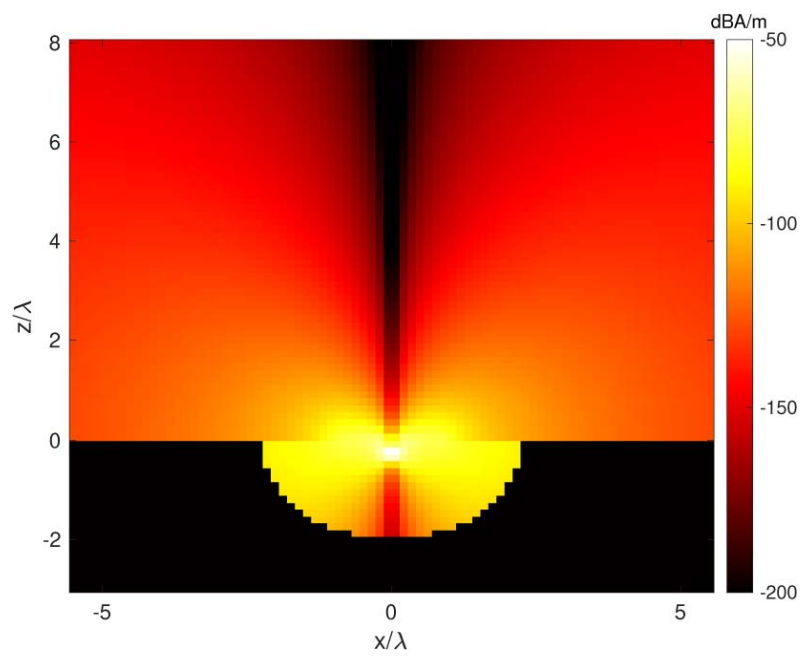

Figure 4. Magnitude of the total magnetic field due to an electric dipole in layer 1 for the DNG-DPS cavity.

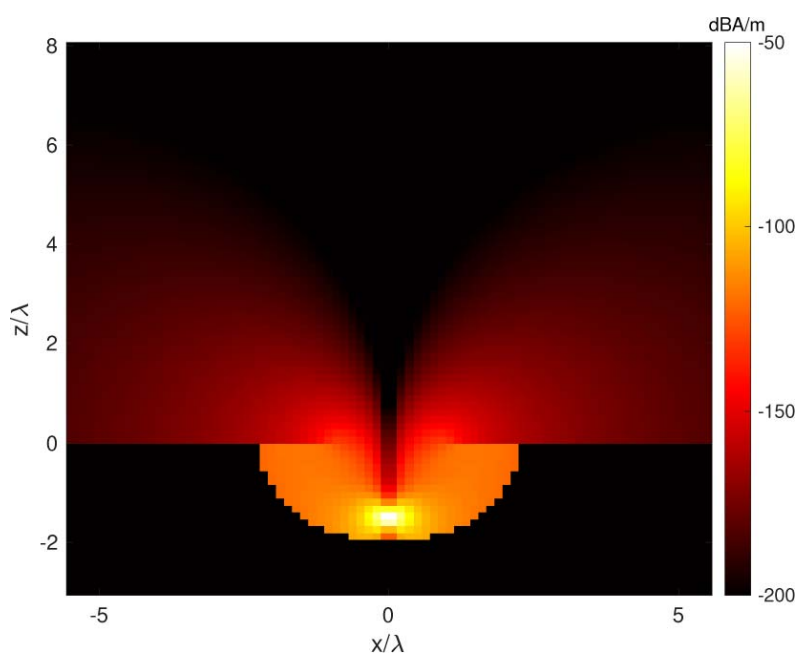

Figure 5. Magnitude of the total magnetic field by an electric dipole in layer 2 for the DPS-DNG cavity. 


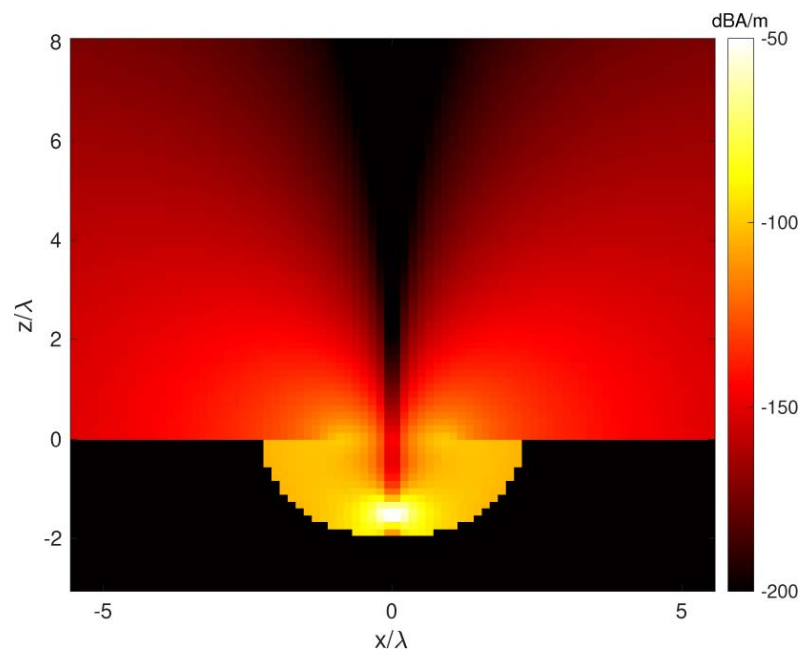

Figure 6. Magnitude of the total magnetic field by an electric dipole in layer 2 for the DNG-DPS cavity.

due to a magnetic dipole, with incident magnetic Hertz vector $\pm \hat{z} \exp (i k R) /(k R)$, at various locations and for different material combinations are given in Figures 7-10.

\section{Conclusion}

This new exact analytical solution for electric and magnetic dipoles radiating inside a two-layered cavity involving anti-isorefractive materials provides one additional benchmark for the validation of computational software [30].

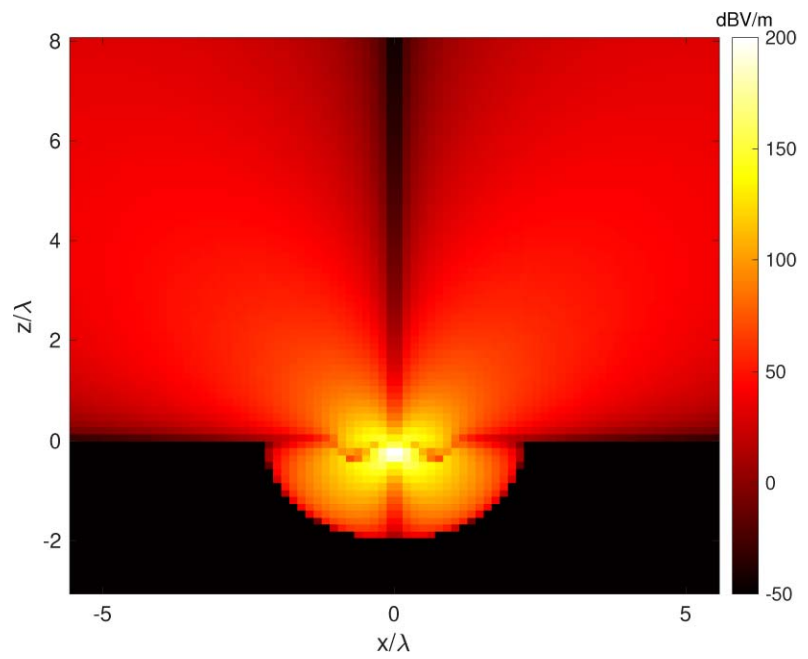

Figure 7. Magnitude of the total electric field by a magnetic dipole in layer 2 for the DPS-DNG cavity.

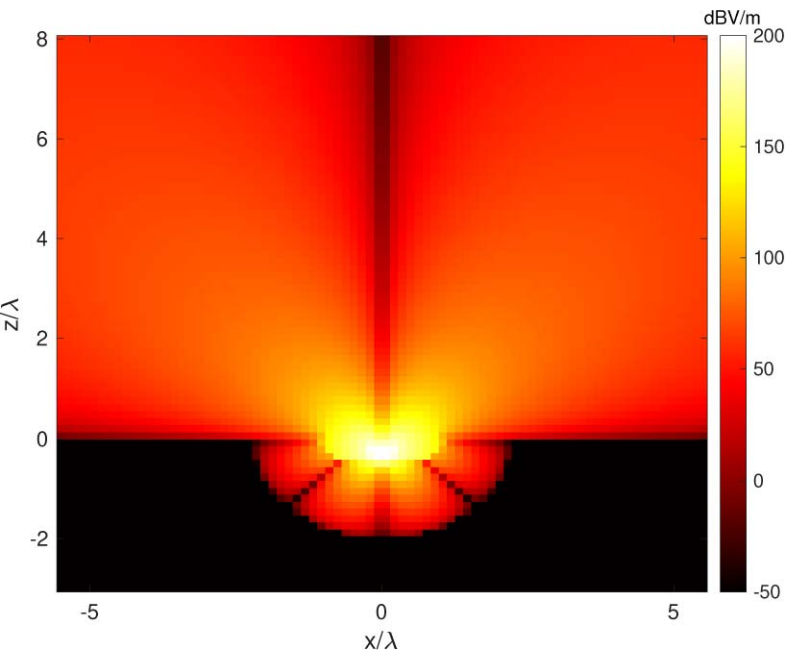

Figure 8. Magnitude of the total electric field by a magnetic dipole in layer 1 for the DNG-DPS cavity.

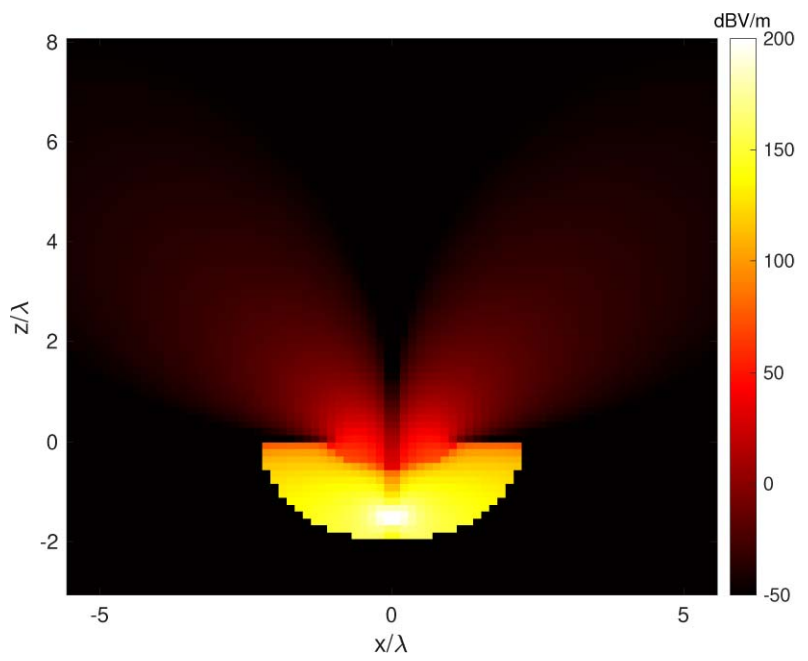

Figure 9. Magnitude of the total electric field by a magnetic dipole in layer 2 for the DPS-DNG cavity.

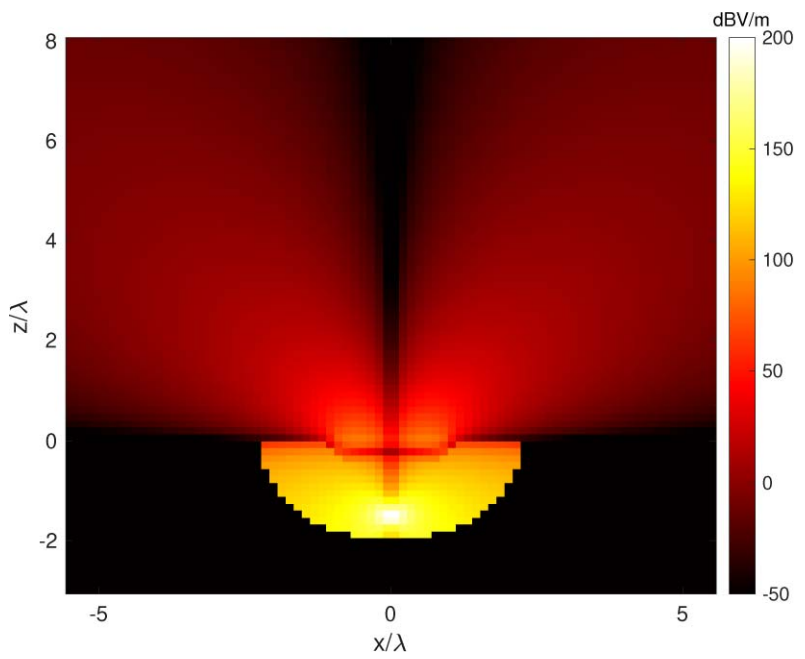

Figure 10. Magnitude of the total electric field by a magnetic dipole in layer 2 for the DNG-DPS cavity. 


\section{References}

1. D. Erricolo, "Acceleration of the Convergence of Series Containing Mathieu Functions Using Shanks Transformation," IEEE Antennas and Wireless Propagation Letters, 2, 2003, pp. 58-61.

2. J. J. Bowman, T. B. A. Senior, and P. L. E. Uslenghi, Electromagnetic and Acoustic Scattering by Simple Shapes, New York, Hemisphere Publishing Corporation, 1987.

3. P. Uslenghi, "Exact Scattering by Isorefractive Bodies," IEEE Transactions on Antennas and Propagation, 45, 9, September 1997, pp. 1382-1385.

4. D. Erricolo, "Isorefractivity: Teaching and Research Perspectives," IEEE Antennas and Propagation Society International Symposium and North American Radio Science Meeting, Vancouver, BC, Canada, July 19-25, 2015.

5. R. Scharstein and A. Davis, "Time-Domain ThreeDimensional Diffraction by the Isorefractive Wedge," IEEE Transactions on Antennas and Propagation, 46, 8, August 1998, pp. 1148.1158.

6. V. Daniele and P. Uslenghi, "Closed-Form Solution for a Line Source at the Edge of an Isorefractive Wedge," IEEE Transactions on Antennas and Propagation, 47, 4, April 1999, pp. 764-765.

7. P. L. E. Uslenghi, "Exact Geometrical Optics Scattering From a Right-Angle Isorefractive Wedge Structure," IEEE Antennas and Wireless Propagation Letters, 3, December 2004, pp. 127-128.

8. P. Uslenghi, "Exact Penetration, Radiation and Scattering for a Slotted Semielliptical Channel Filled With Isorefractive Material," IEEE Transactions on Antennas and Propagation, 52, 6, June 2004, pp. 1473-1480.

9. D. Erricolo, M. D. Lockard, C. M. Butler, and P. L. E. Uslenghi, "Numerical Analysis of Penetration, Radiation, and Scattering for a 2D Slotted Semielliptical Channel Filled With Isorefractive Material," Progress in Electromagnetics Research, 53, 2005, pp. 69-89.

10. D. Erricolo, M. Lockard, C. Butler, and P. Uslenghi, "Currents on Conducting Surfaces of a SemiellipticalChannel-Backed Slotted Screen in an Isorefractive Environment," IEEE Transactions on Antennas and Propagation, 53, 7, July 2005, pp. 2350-2356.

11. D. Erricolo and P. L. E. Uslenghi, "Penetration, Radiation, and Scattering for a Cavity-Backed Gap in a Corner," IEEE Transactions on Antennas and Propagation, 53, 8, August 2005, pp. 2738-2748.

12. D. Erricolo and P. L. E. Uslenghi, "Exact Radiation and Scattering for an Elliptic Metal Cylinder at the Interface Between Isorefractive Half-Spaces," IEEE Transactions on Antennas and Propagation, 52, 9, September 2004, pp. 2214-2225.

13. D. Erricolo, P. Uslenghi, B. Elnour, and F. Mioc, "Scattering by a Blade on a Metallic Plane," Electromagnetics, 26, 1, January 2006, pp. 57-71.

14. M. Valentino and D. Erricolo, "Exact Two-Dimensional Scattering From a Slot in a Ground Plane Backed by a Semielliptical Cavity and Covered With an Isorefractive Diaphragm," Radio Science, 42, RS6S12, November 2006, doi:10.1029/2006RS003547.

15. S. Canta and D. Erricolo, "Exact 2D Scattering Analysis of a Slot Backed by a Semielliptical Cavity and Covered by a Multilayer Diaphragm," Radio Science, 43, December 2008, doi:10.1029/2007RS003809.
16. O. Akgol, D. Erricolo, and P. Uslenghi, "Exact Imaging by an Elliptic Lens," IEEE Antennas and Wireless Propagation Letters, 10, June 2011, pp. 639-642.

17. O. Akgol, D. Erricolo, and P. Uslenghi, "Electromagnetic Radiation and Scattering for a Gap in a Corner Backed by a Cavity Filled With DNG Metamaterial," Radio Science, 46, RS4005, August 2011, doi:10.1029/2010RS004471.

18. O. Akgol, V. Daniele, D. Erricolo, and P. Uslenghi, "Radiation From a Line Source Shielded by a Confocal Elliptic Layer of DNG Metamaterial," IEEE Antennas and Wireless Propagation Letters, 10, August 2011, pp. 943-946.

19. J. Liang and P. Uslenghi, "Exact Scattering by Isorefractive Paraboloidal Radomes," IEEE Transactions on Antennas and Propagation, 55, 6, June 2007, pp. 15461553.

20. S. Roy and P. Uslenghi, "Exact Scattering for Axial Incidence on an Isorefractive Paraboloid," IEEE Transactions on Antennas and Propagation, 45, 10, October 1997, p. 1563.

21. P. L. E. Uslenghi, "On the Sharpness of a Needle [Electromagnetic Propagation]," IEEE Antennas and Propagation Magazine, 46, 2, April 2004, pp. 146-147.

22. C. Berardi, D. Erricolo, and P. L. E. Uslenghi, "Exact Dipole Radiation for an Oblate Spheroidal Cavity Filled With Isorefractive Material and Aperture-Coupled to a Half Space," IEEE Transactions on Antennas and Propagation, 52, 9, September 2004, pp. 2205-2213.

23. D. Erricolo and P. Uslenghi, "Exact Radiation for Dipoles on Metallic Spheroids at the Interface Between Isorefractive Half-Spaces," IEEE Transactions on Antennas and Propagation, 53, 12, December 2005, pp. 3974-3981.

24. M. Valentino and D. Erricolo, "Exact Radiation of a Dipole in the Presence of a Circular Aperture in a Ground Plane Backed by a Spheroidal Cavity and Covered With an Isorefractive Diaphragm," Radio Science, 42, rS6S13, December 2007, doi:10.1029/2006RS003548.

25. T. Negishi, D. Erricolo, and P. L. E. Uslenghi, "Metamaterial Spheroidal Cavity to Enhance Dipole Radiation," IEEE Transactions on Antennas and Propagation, 63, 6, June 2015, pp. 2802-2807.

26. Y. Liu, T. Negishi, and D. Erricolo, "Exact Electromagnetic Scattering From a Dipole Antenna Located Inside a Multilayer Metamaterial Oblate Spheroidal Cavity," USNC-URSI National Radio Science Meeting, Boulder, CO, USA, January 6-9, 2016.

27. A. N. Askarpour and P. L. E. Uslenghi, "Exact Dipole Radiation From an Oblate Semi-Spheroidal Cavity Filled With DNG Metamaterial," IEEE Transactions on Antennas and Propagation, 59, 7, July 2011, pp. 2473-2479.

28. C. Flammer, Spheroidal Wave Functions, Stanford, CA, Stanford University Press, 1957.

29. D. Erricolo and T. Negishi, "Symmetry Properties of the Spheroidal Functions With Respect to Their Parameter," IEEE Transactions on Antennas and Propagation, 65, 9, September 2017, pp. 4947-4951.

30. D. Erricolo, "Benchmarking Computational Electromagnetics With Exact Analytical Solutions of Canonical Electromagnetic Scattering Problems," IEEE Antennas and Propagation Society International Symposium/ USNC-URSI National Radio Science Meeting, San Diego, CA, USA, July 9-14, 2017. 